\title{
Hybrid Exotic Meson Decay Width
}

\author{
M.S. Cook* and H.R. Fiebig, LHP Collaboration \\ Physics Department \\ Florida International University \\ Miami, Florida 33199 USA \\ E-mail: mcook003efiu.edu
}

\begin{abstract}
We present results of a decay width calculation for a hybrid exotic meson $\left(h, J^{P C}=1^{-+}\right)$in the decay channel $h \rightarrow \pi a_{1}$. This calculation uses quenched lattice QCD and Lüscher's finite box method. Operators for the $h$ and $\pi a_{1}$ states are used in a correlation matrix which was expanded by varying the smearing and fuzzing levels at source and sink points. Scattering phase shifts for a discrete set of relative $\pi a_{1}$ momenta are determined using eigenvalues of the correlation matrix and formulae derived by Lüscher. The phase shift data is very sparse, but fits to a Breit-Wigner model are made, resulting in a decay width of about $80 \mathrm{MeV}$.
\end{abstract}

XXIIIrd International Symposium on Lattice Field Theory

25-30 July 2005

Trinity College, Dublin, Ireland

\footnotetext{
${ }^{*}$ Speaker.
}

†This material is based upon work supported by the U.S. National Science Foundation under Grant No. PHY0300065 and upon resources provided by the Lattice Hadron Physics Collaboration(LHPC) through the SciDac program of the U.S. Department of Energy 


\section{Introduction}

Most states in the known hadron spectrum are resonances. Their decay widths, arguably more so than their masses, are sensitive indicators of the intrinsic structure and forces between the constituents. The decay of molecule-like states, for example, may be driven by a residual (effective) force and thus give rise to widths typical of nuclear physics. Spatially more compact hadrons could follow a quite different mechanism.

Decays can be studied with lattice techniques [1]. In particular, lattice QCD provides us with $a b$ initio access to unusual hadrons, like hybrids and exotics, which are a focus of some experimental programs currently under way. We here give an overview about a quenched lattice simulation set up to estimate the decay width of a $J^{P C}=1^{-+}$hybrid exotic meson resonance, say $h$, coupled to a $\pi a_{1}$ decay channel. Correlation matrices based on the corresponding operators yield (excited) mass spectra. Lüscher's finite box method [2,3] is then applied to get the scattering phase shifts at a discrete set of relative $\pi a_{1}$ momenta. A decay width is determined by fitting Breit-Wigner functions.

Such a simulation is viable, on a reasonably sized lattice, if the energy spectrum exhibits a level crossing between states excited by $h$ and $\pi a_{1}$ operators, preferably, close to the physical value of the pion mass. Unlike in $\rho \rightarrow \pi \pi$, where this is very hard to achieve, $h \rightarrow \pi a_{1}$ allows a relative $s$-wave in the decay channel and may thus proceed with relative lattice momentum zero. The feasibility of the current work much relies on this fact.

\section{Lattice simulation}

Hybrid mesons are quark-antiquark pairs having valence gluons as structural components. Following [4] our choice for the hybrid operator is

$$
O_{h^{+} ; j}(t)=\frac{1}{\sqrt{V}} \sum_{\vec{x}} \sum_{i=1}^{3} \bar{d}_{a}(\vec{x} t) \gamma_{i} u_{b}(\vec{x} t)\left(F_{i j}^{a b}(\vec{x} t)-F_{i j}^{\dagger a b}(\vec{x} t)\right) .
$$

Here $a, b$ are color indices and $F_{i j}$ is a product of SU(3) link matrices defined on paths forming a clover leaf in the $i-j$ plane with center at $\vec{x}$, and $V$ is the spatial lattice volume. The quantum numbers are $I=1$ and $J^{P C}=1^{-+}$, where the combination $F_{i j}-F_{i j}^{\dagger}$ is needed for positive charge conjugation. Appropriate operators for $\pi a_{1}$ can be constructed from

$$
O_{\pi^{+} a_{1}^{0} ; j}(t)=\frac{1}{\sqrt{V}} \sum_{\vec{x}} \sum_{\vec{y}} \delta_{\vec{y}-\vec{x}, \vec{r}} \bar{d}(\vec{x} t) \gamma_{5} u(\vec{x} t) \bar{d}(\vec{y} t) \gamma_{5} \gamma_{j} d(\vec{y} t)
$$

The simplest spatial configuration for an $s$-wave $\left(A_{1}\right.$ irrep) is the choice $\vec{r}=0$, which we use in this work. The elements in the resulting correlation matrix,

$$
C\left(t, t_{0}\right)=\left(\begin{array}{cc}
C_{h, h}\left(t, t_{0}\right) & C_{h, \pi a_{1}}\left(t, t_{0}\right) \\
C_{\pi a_{1}, h}\left(t, t_{0}\right) & C_{\pi a_{1}, \pi a_{1}}\left(t, t_{0}\right)
\end{array}\right),
$$

are understood to include spin traces, for example $C_{h, h}\left(t, t_{0}\right)=\sum_{j=1}^{3}\left\langle O_{h^{+} ; j}(t) O_{h^{+} ; j}^{\dagger}\left(t_{0}\right)\right\rangle$, and so on.

The flavor structure of the operator (2.2) is $\pi a_{1} \sim \bar{d} u \bar{d} d$, which gives rise to (two) equal time contractions $\overline{\bar{d}(t) d}(t)$ in (2.3). To avoid having to compute those propagator elements we observe 


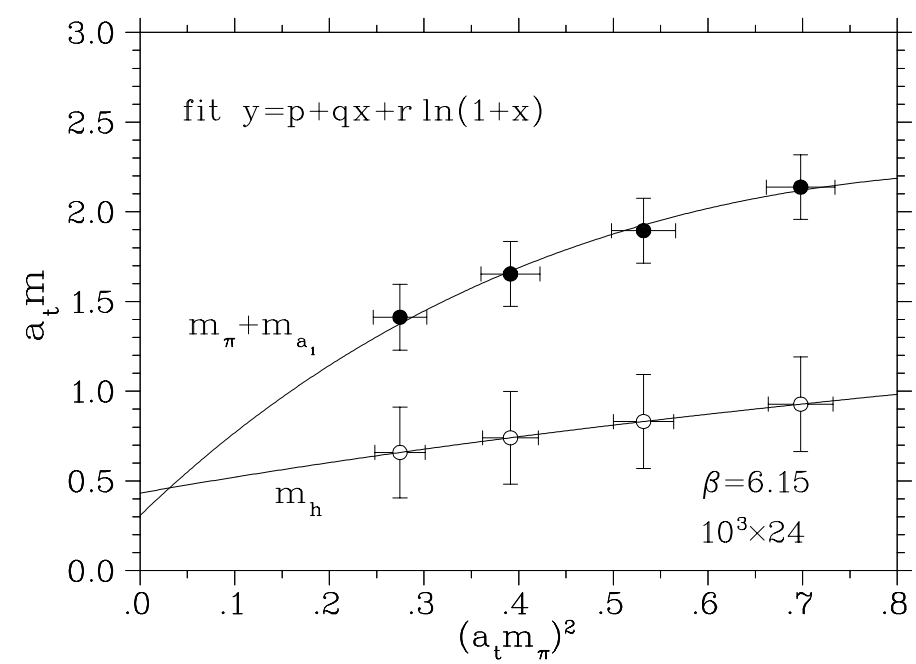

Figure 1: Masses of the $h$ and $\pi+a_{1}$ non-interacting system versus the pion mass squared. The extrapolation shows an energy level crossing at light pion mass. If the physical scale is set to the $a_{1}$ meson mass, 1.26 $\mathrm{GeV}$, then the hybrid mass extrapolates to $1.73 \pm .39 \mathrm{GeV}$. If the $\rho$ meson sets the scale, then the hybrid mass extrapolates to $1.46 \pm .31 \mathrm{GeV}$.

that these do not occur for $\pi K_{1} \sim \bar{d} u \bar{d} s$. Since the experimental $a_{1}$ and $K_{1}$ masses are very close, $1260 \mathrm{MeV}$ and $1270 \mathrm{MeV}$ respectively, and given that only the mass spectrum is input to Lüscher's method we argue that neglecting equal time contractions in $C_{\pi a_{1}, \pi a_{1}}$ should not significantly effect our results. It should be emphasized that this approximation only is applied to $C_{\pi a_{1}, \pi a_{1}}$ in (2.3), the off-diagonal correlators can be computed or inferred using hermiticity.

Wuppertal style smearing is done on the quark fields, using APE style gauge link fuzzing in the process. We use a common strength factor $(\alpha=2.5)$ and either 1,2 , and 3 smearing iterations. The same smearing is done at source and sink points. In this way the correlator (2.3) expands to a $6 \times 6$ hermitian matrix

$$
C\left(t, t_{0}\right)_{2 \times 2} \longrightarrow \mathscr{C}\left(t, t_{0}\right)_{6 \times 6} .
$$

Simulations are made using the anisotropic Wilson gauge action with bare aspect ratio $a_{s} / a_{t}=$ 2 on lattice sizes $L^{3} \times 24, L=8,10,12$, at $\beta=6.15$, and the corresponding Wilson fermion action in quenched approximation. Four hopping parameters, $\kappa=0.140,0.136,0.132,0.128$, were used with a multiple mass solver [5]. Figure 1 shows the ground state masses of the $h$ and the $\pi+a_{1}$ systems versus $m_{\pi}^{2}$. These are obtained using effective mass functions based on output of individual $h, \pi$, and $a_{1}$ correlators at smearing level two. The three parameter fit model, see inset in Fig. 1, matches most of the energy-versus- $m_{\pi}^{2}$ data produced in this work. Its choice is purely empirical. Setting the physical scale to the $\rho$ meson mass results in a lattice constant of $a_{t}=0.07 \mathrm{fm}$, and this value is used consistently for the width calculations.

\section{Analysis}

To extract the mass spectrum the $6 \times 6$ correlation matrix (2.4) is first diagonalized on a fixed timeslice $t_{1}$,

$$
\mathscr{C}\left(t_{1}, t_{0}\right)=V\left(t_{1}, t_{0}\right) D\left(t_{1}, t_{0}\right) V^{\dagger}\left(t_{1}, t_{0}\right)
$$



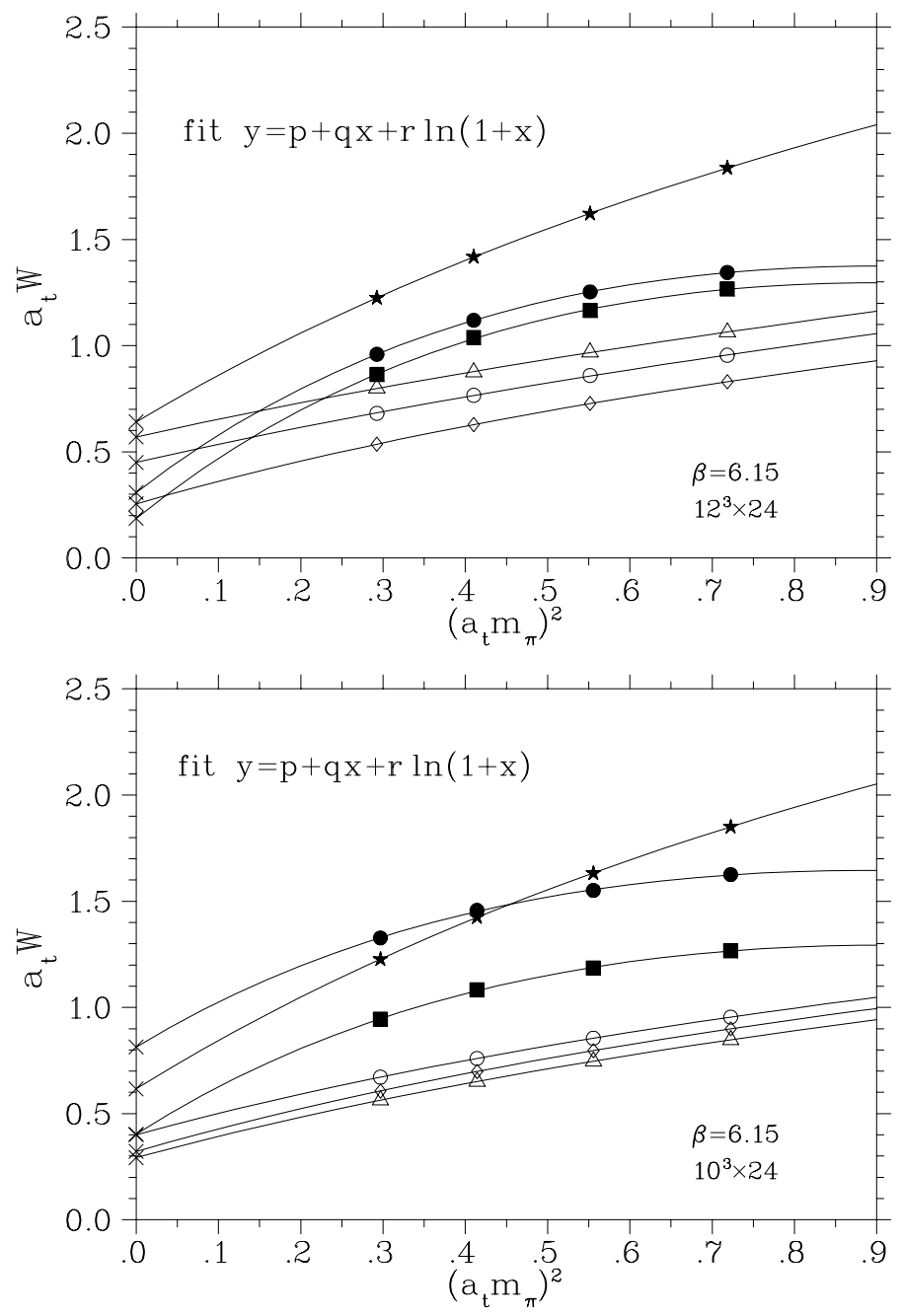

Figure 2: Energy spectra $W_{n}, n=1 \ldots 6$, and extrapolation to small pion mass for the $12^{3} \times 24$ and $10^{3} \times 24$ lattices. Error bars are omitted for clarity.

where $V\left(t_{1}, t_{0}\right)$ is unitary and $D\left(t_{1}, t_{0}\right)$ is diagonal and positive definite. To ensure the latter $t_{1}$ should be an 'early' timeslice, we here use $t_{1}-t_{0}=4$. The correlation matrix is then subject to a basis transformation and normalization according to

$$
\Lambda(t)=\frac{1}{\sqrt{D\left(t_{1}, t_{0}\right)}} V^{\dagger}\left(t_{1}, t_{0}\right) \mathscr{C}\left(t, t_{0}\right) V\left(t_{1}, t_{0}\right) \frac{1}{\sqrt{D\left(t_{1}, t_{0}\right)}} .
$$

This procedure amounts to solving the usual generalized eigenvalue problem. The only modification being that the eigenvectors (columns of $V$ ) used for the basis transformation in (3.2) are employed at a fixed timeslice. Thus we find that $\Lambda(t)$ is approximately diagonal. We will simply refer to the diagonal elements, $\Lambda_{n n}(t)$, as the eigenvalues, $\lambda_{n}(t)$. Compared to 'diagonalizing at all $t$ ' statistical fluctuations are reduced. The eigenvalues $\lambda_{n}(t), n=1 \ldots 6$, give rise to effective mass functions from which the spectral energies $W_{n}$ are obtained. Energy spectra for the $12^{3} \times 24$ and $10^{3} \times 24$ lattices are shown in Fig. 2 . 
With the spectra known, subsets of masses $W_{n}$ are selected that fall within the elastic region,

$$
\left(m_{\pi}+m_{a_{1}}\right)<W_{n}<2\left(m_{\pi}+m_{a_{1}}\right)
$$

All masses in (3.3) are taken at each of the four values of $m_{\pi}$ and in the limit $m_{\pi} \rightarrow 0$ using the extrapolation. For those sets the relativistic dispersion relation

$$
W_{n}=\sqrt{m_{\pi}^{2}+k_{n}^{2}}+\sqrt{m_{a_{1}}^{2}+k_{n}^{2}}
$$

is solved for the relative $\pi a_{1}$ momenta $k_{n}$. Note, $k_{n}$ is a prediction for the relative momentum of a $\pi a_{1}$ system and is not subject to lattice discretization. These momenta are input to Lüscher's formula [2] for the s-wave scattering phase shifts $\delta_{n}$,

$$
\tan \delta_{n}=-\frac{\pi^{3 / 2} q_{n}}{\mathscr{Z}\left(1 ; q_{n}^{2}\right)} \quad, \quad q_{n}=\frac{k_{n} L_{s}}{2 \pi} .
$$

Here $\mathscr{Z}\left(1 ; q^{2}\right)$ is a generalized $\zeta$-function, and $L_{s}=L a_{s}$ is the physical size of the spatial box (using the bare anisotropy, $a_{s}=2 a_{t}$ ). We then attempt fits to all sets of phase shift data with a Breit-Wigner model,

$$
\tan \delta(k)=\frac{\Gamma / 2}{E_{0}-W(k)} \quad, \quad \text { with } \quad W(k)=\sqrt{m_{\pi}^{2}+k^{2}}+\sqrt{m_{a_{1}}^{2}+k^{2}}
$$

where $E_{0}$ and $\Gamma$ are parameters. When the fit is successful, $\Gamma$ may be interpreted as a decay width. In Fig. 3 we show selected results for the scattering phase shift data and the corresponding BreitWigner fits. The scattering phase angle data does not alone justify the use of Breit-Wigner functions. Use of these functions requires one to assume a priori that a resonant condition exists.

\section{Result}

Physical decay widths, found by using extrapolated energy spectra, varied from $78 \pm 28 \mathrm{MeV}$ to $88 \pm 23 \mathrm{MeV}$ for the three lattice volumes tested. A large portion of the statistical error came from the hybrid correlator. As shown in Fig. 3, the phase shift data is very sparse. The best fits to the Breit-Wigner model returned a resonance energy of $E_{0} \approx 1.9 \mathrm{GeV}$.

Phase shift data obtained using non-extrapolated spectra did not match up well to the BreitWigner model. The reason being, as shown in Fig. 1, the pion masses used are quite far from the actual energy level crossing, and thus, one is less likely to observe resonance behavior.

\section{Summary}

Using Lüscher's method, a decay width for the $1^{-+}$hybrid exotic meson is calculated to be about $80 \mathrm{MeV}$ with a statistical error of $25 \mathrm{MeV}$. The number of data points available to fit BreitWigner functions is sparse. There is a definite energy level crossing between the $h$ and $\pi a_{1}$ system at light pion mass giving credence to the simulation. 

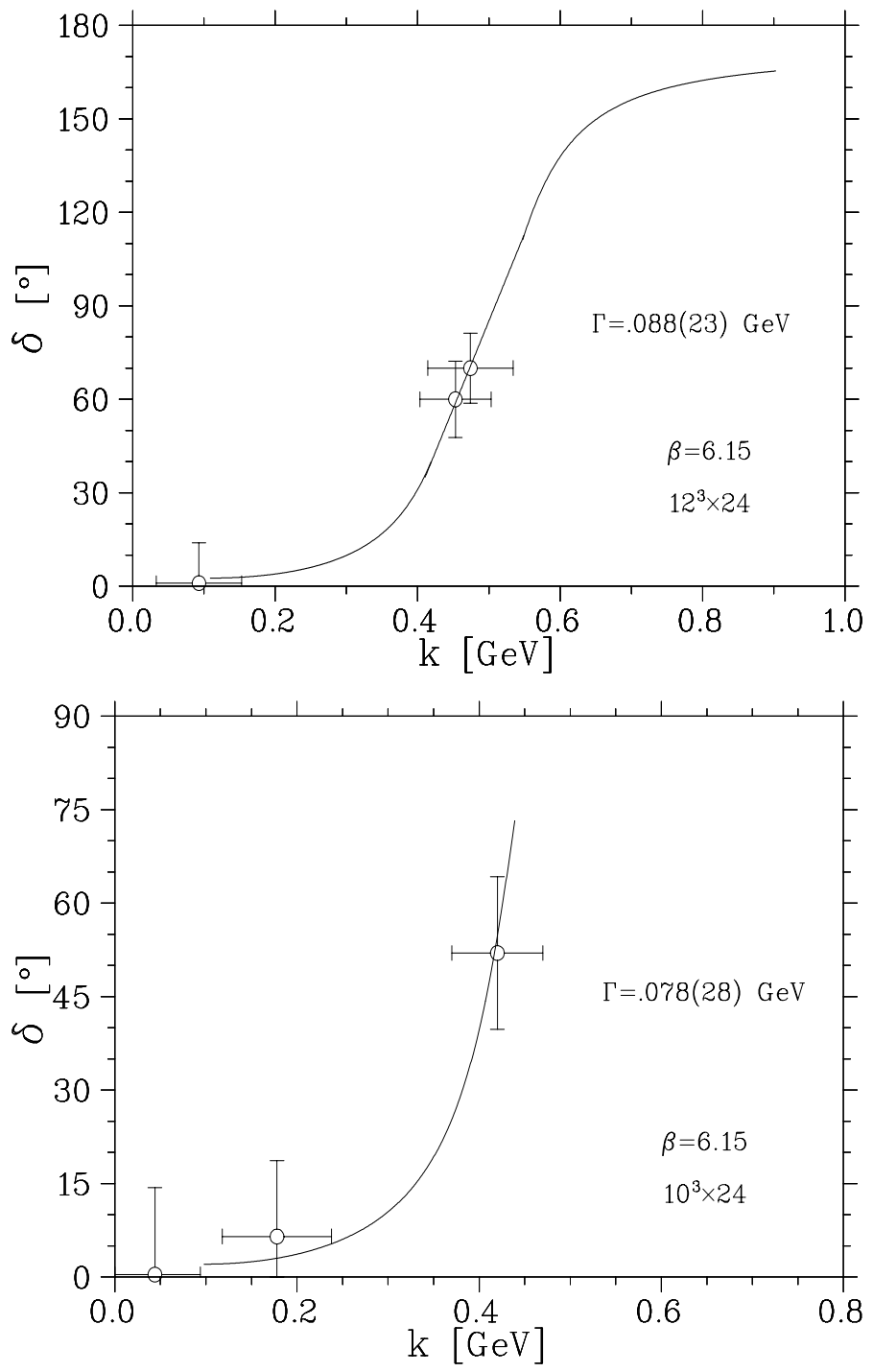

Figure 3: S-wave scattering phase shift versus momentum for the $12^{3} \times 24$ and $10^{3} \times 24$ lattices using extrapolated spectra from Fig. 2. The curve fits are Breit-Wigner models (3.6), giving widths of $88 \pm 23$ $\mathrm{MeV}$ and $78 \pm 28 \mathrm{MeV}$ respectively.

\section{References}

[1] C. Michael. Hadronic decays. In proceedings of The 23rd International Symposium on Lattice Field Theory, 25-30 July 2005, Trinity College, Dublin, Ireland, PoS(LAT2005)008, [hep-lat/0509023].

[2] M. Lüscher. Signatures of unstable particles in finite volume. Nucl. Phys., B364:237-254, 1991.

[3] M. Lüscher. Two particle states on a torus and their relation to the scattering matrix. Nucl. Phys., B354:531-578, 1991.

[4] C. Bernard et al. Exotic mesons in quenched lattice qcd. Phys. Rev., D56:7040-7041, 1997.

[5] U. Glässner, S. Güsken, T. Lippert, G. Ritzenhöfer, K. Schilling and A. Frommer, "How to compute Green's functions for entire mass trajectories within Krylov solvers", Int. J. Mod. Phys., C7(1996)635, [hep-lat/9605008]. 